\title{
Pole-Movement in Oscillators and Energy Balance Principle
}

\author{
$\underline{\text { S. Pal }}^{\text {, S. Chatterjee }}{ }^{2}$, and B.N. Biswas ${ }^{1}$ \\ ${ }^{1}$ Sir J C Bose School of Engineering, SKF Group of Institutions, Hooghly 712 139, West Bengal, INDIA, \\ subhradeeppal@gmail.com and baidyanathbiswas@gmail.com \\ ${ }^{2}$ Kanailal Vidyamandir (Fr. Section), Chandannagar, Hooghly 712 136, West Bengal INDIA, somnathchat@yahoo.com
}

\begin{abstract}
(1) How do the poles behave in soft-self and hard-self oscillators executing a process of transition from the initial state to the final state and distortion is a desired contamination, on the complex frequency plane? (2) Do the poles ultimately reside on the imaginary axis of the complex frequency plane? (3) If the poles do not reside on the imaginary axis, what is the frequency of oscillation? (4) In such a non-steady non-linear environment where both the frequency and amplitude of oscillation are changing, then how to apply Barkhausen criteria to study oscillation growth? (5) Can we invoke the Energy Balance principle in such situations? These are some of the questions that this paper deals with.
\end{abstract}

\section{Introduction}

"For a sinusoidal oscillator circuit to start producing sinusoidal output, the poles of the circuit must be initially situated in the right half plane and some mechanism must exist that moves these poles to the imaginary axis, while the amplitude grows" $[1,2,3]$. Apparently it is assumed that a (soft) self-excited oscillator in the steady state behaves like an ideal linear mathematical harmonic lossless oscillator obeying the second order differential equation, with a pair of poles on the imaginary axis of the complex frequency plane. It oscillates with amplitude equal to that given as excitation. But in a self-excited oscillator the oscillation has to build up from an insignificant excitation and ultimately to attain a desired steady state value. In a hard self-excited oscillator also oscillation builds up from a threshold value of excitation to a steady state. This paper attempts to clarify these questions.

\section{System Equation and Modified Barkhausen Criteria}

Refer to Op Amp Wien Bridge Oscillator as shown in Fig. 1. Different node voltages are also shown and the corresponding circuit equations can be written as

$$
i_{a} R+\frac{1}{C} \int i_{a} d t=v_{0}(v)+v_{i}-v
$$

Note that the output voltage is a function of the input voltage ' $v$ ' and ' $v_{i}$ ' is a signal generated by the inherent noise or switching of the systems. The from (1) and referring to Fig.1 one can write

$$
R C \frac{d v}{d t}+\left[3 v-v_{0}(v)-v_{i}\right]+\frac{1}{R C} \int v d t=0
$$

In an ideal situation, $v_{0}(v)=G v$. Thus if it is possible to have $\mathrm{G}=3$, then $\mathrm{W}$. B. Oscillator would turn into an ideal harmonic one having poles on the imaginary axis. Note that because of the non linear nature of the governing equation (2) it is expected that the instantaneous voltage ' $v$ ' will be of the form:

$$
v(t)=A(t) \exp \left[j \omega_{1} t+\theta(t)\right]
$$

So we define the instantaneous frequency as

$$
j \omega=\frac{1}{v(t)} \cdot \frac{d v}{d t}=\frac{1}{A} \frac{d A}{d t}+j \omega_{1}+j \frac{d v}{d t}
$$

Therefore, (2) can be expressed as $(s=j \omega)$ :

$$
\frac{v}{v_{i}}=\frac{G(s)}{1-G(s) N(A)}
$$

Where

$$
\omega_{0}=1 / R C \text { and } \frac{1}{G(s)}=\left(\frac{s}{\omega_{0}}+3+\frac{\omega_{0}}{s}\right)
$$


Here $N(A)$ is the equivalent linearised gain of the linearity between the input and the output $v_{0}(v)$ vs. $v$. The block diagrammatic representation is shown in Fig. 2. Therefore modified Barkhausen condition of oscillation is given by

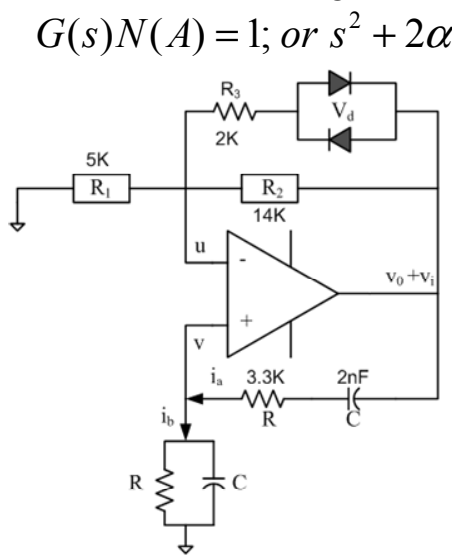

Fig. 1 Wien Bridge Oscillator

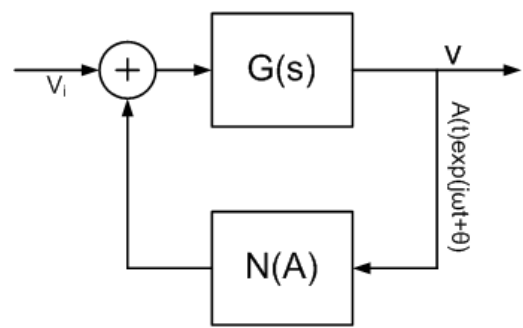

Fig.2: Barkhausen's Feedback Model with Nonlinearity and Nonsteady Situation

This relation (7) takes into account both non-steady state situation and non-linearity of the circuit. These were the limitations of Barkhausen criterion. Incidentally, these limitations have been overcome by invoking the quasi-static principle. That is, the entire time domain observation has been considered as a sum total of a large number of small intervals, and in each of the small time interval the gain of the circuit is assumed to remain constant. The value of the corresponding gain is in this small time interval is determined by using the principle of describing function technique $N(A)$. Equation (7) specifies the location of the poles. The value of $N(A)$ and hence $\alpha(A)$ can be evaluated easily by referring to the input-output relation for this particular experimental circuit, viz.

$$
\begin{aligned}
& v_{0}(v)=4.0188 v-3.6145 v^{2}-10.386 v^{3}=\alpha_{1} v-\beta v^{2}-\gamma v^{3} \\
& N(A)=\left[4.0188-\frac{3}{4} .(10.38) A^{2}\right] ; \text { hence } 2 \alpha(A)=-1.0188+7.785 A^{2}
\end{aligned}
$$

From (7) one finds that the poles are

\section{Growth of Oscillation and Pole Movement}

$$
s_{1,2}=-\alpha(v) \omega_{0} \pm j \omega_{0} \sqrt{1-\alpha^{2}(v)}
$$

Refer to Fig.3 that plots the variation of the damping factor against the instantaneous amplitude coupled with the buildup of oscillations. From the figure it is at once evident that when the input is small and $|v|<\sqrt{ }(1.0188 / 7.785)=361 \mathrm{mV}$, the oscillation grows because $\alpha(v)$ is negative. Since the amplitude continues to grow and this means that $\alpha(v)$ becomes positive signifying delay of the amplitude. It is obvious that changing of sign of the damping factor means migration of the poles from the right half plane to the left half plane of the complex frequency plane. And as soon as $|v|<361 \mathrm{mV}$ the oscillation again grows and so the poles move again to the right half plane. This back and forth movement is due to the following mechanism. When the poles are the right half plane the oscillator draws energy from the battery and when the poles moves to the left half plane the oscillator dissipates. It can be used to calculate the average steady state (so to say) value of the amplitude of oscillation by applying Energy Balance Technique as illustrated in the following.

Referring to (2) and (8) one finds that

\section{Energy Balance Technique and Damping Factor}

$$
\frac{d^{2} v}{d t^{2}}+\omega_{0} \frac{d}{d t}\left[\alpha v-\beta v^{2}-\gamma v^{3}\right]+\omega_{0}^{2} v=0 \quad \text { where, } \quad \alpha=3-\alpha_{1}
$$

One can rewrite the oscillation equation (11) as

$$
\frac{d^{2} x}{d t^{2}}-\alpha\left[1-\frac{2 \beta \sqrt{\gamma}}{\sqrt{\alpha}} x-x^{2}\right] \frac{d x}{d t}+x=0 \quad \text { where, } \quad \omega_{0} t=\tau, x=v / v_{0}
$$


We define an energy function $\mathrm{E}$ as

$$
E=\frac{1}{2}(\dot{x})^{2}+\frac{1}{2}(x)^{2} ; \frac{d E}{d t}=\frac{1}{2}(2 \ddot{x} \ddot{x}+2 \dot{x} x)
$$

which on using (12) reduces to

$$
\frac{d E}{d t}=\alpha\left[(\dot{x})^{2}-\left(\beta \cdot x \dot{x}+x^{2} \dot{x}^{2}\right)\right]
$$

Therefore, the average energy variation over a period is

$$
\frac{d\langle E\rangle}{d t}=\alpha\left[\left\langle\dot{x}^{2}\right\rangle-\left(\beta\langle x \dot{x}\rangle+\left\langle x^{2} \dot{x}^{2}\right\rangle\right)\right]
$$

The first term on the right hand side of (15) denotes production of energy where's the second term within the braces denoted the dissipation of energy. In the steady state as explained earlier, the energy generation per cycle becomes equal to the energy dissipation. That is

$$
\left\langle\dot{x}^{2}\right\rangle=\left(\beta\langle x \dot{x}\rangle+\left\langle x^{2} \dot{x}^{2}\right\rangle\right)
$$

Assuming nearly sinusoidal solution, i.e., $x=A_{s} \cos \tau$, it is easily shown that

$$
\begin{gathered}
\left\langle(\dot{x})^{2}\right\rangle=\frac{1}{2 \pi} \int_{t_{0}}^{t+2 \pi} \dot{x}^{2} d t=\frac{1}{2} A_{s}^{2} \\
\left(\beta\langle x \dot{x}\rangle+\left\langle x^{2} \dot{x}^{2}\right\rangle\right)=\frac{A_{s}^{4}}{8} \\
A_{s}^{2}=2
\end{gathered}
$$

Hence from (16), (17) and (18) one finds,
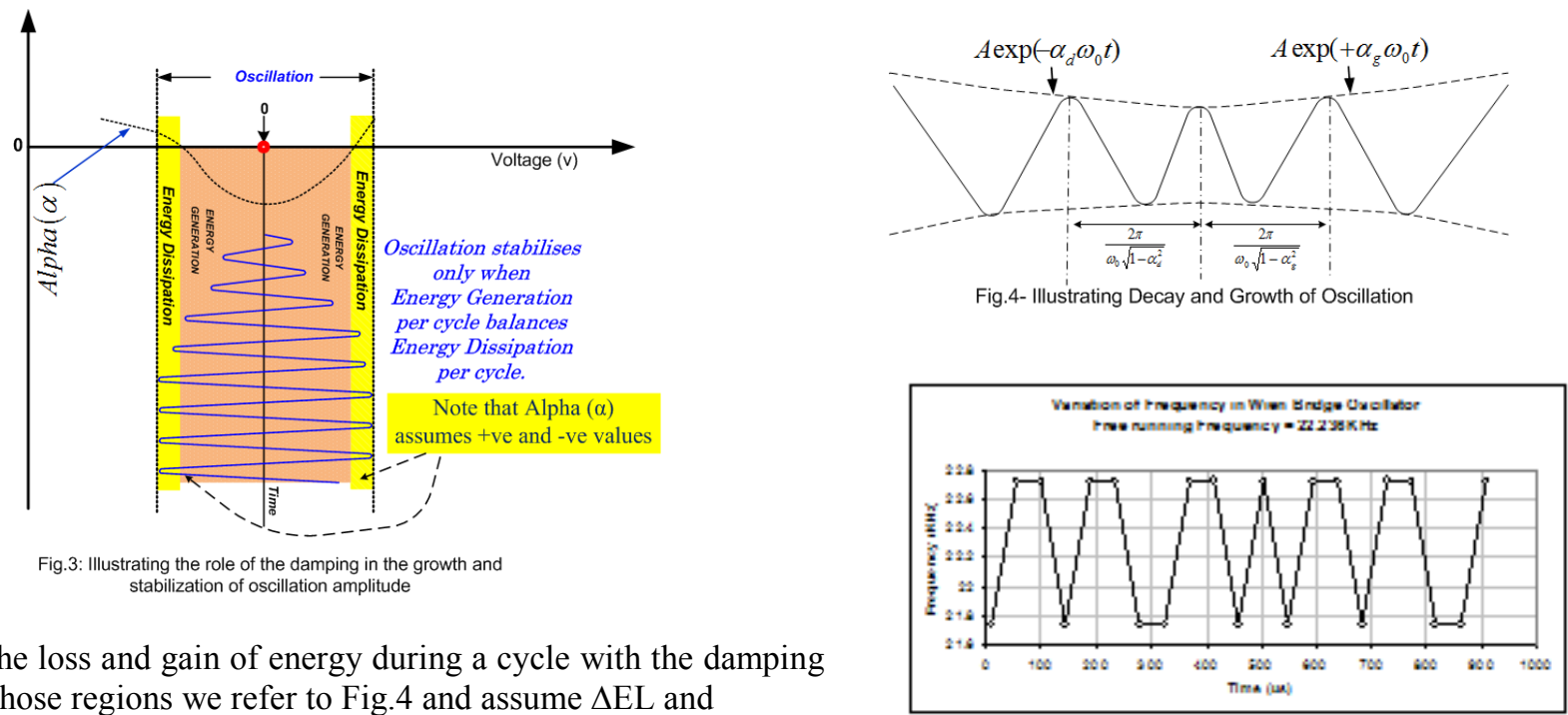

To relate the loss and gain of energy during a cycle with the damping factors in those regions we refer to Fig. 4 and assume $\Delta \mathrm{EL}$ and $\Delta \mathrm{EG}=$ energy lost or gained during one cycle and $\mathrm{E}=$ energy at the beginning of the cycle it is easily shown that [7]:

Fig.5 - Frequency variation with Time

$$
4 \pi \alpha_{L}=\frac{\Delta E L}{E}=\frac{A_{S}^{2} / 2}{A_{S}^{2} / 2} \text { and } 4 \pi \alpha_{G}=\frac{\Delta E G}{E}=\frac{A_{S}^{4} / 8}{A_{S}^{2} / 2}
$$

Assuming that the average amplitudes during the decaying cycle and growing cycle are nearly equal then from (19) and (20) one gets easily $\alpha_{\mathrm{L}}=1 / 2 \pi$ and $\alpha_{\mathrm{G}}=-A^{2} /(4.2 \pi)$. "A" may be taken as " $\mathrm{A}_{\mathrm{s}}$ " approximately. This clearly indicates that during oscillation; poles move back and forth between the left half and right half plan of the complex frequency plane. This process repeats. Moreover, the shift to the right half plane is equal to the right half plane. This is shown in Fig. 5 which has been observed experimentally. 


\section{Effect of Distortion on Damping Factor}

Because of necessity of incorporating a limiter type non-linearity within an oscillator in order to check the growth of oscillation, distortion at the output waveform is a desired contamination. In this particular case, the non-linearity is a cubic type and as such that will be their harmonic distortion however small it may be. Therefore the output ' $\mathrm{x}$ ' is of the following form $[4,8]$,

$$
x=A \cos (\tau)-b \sin (3 \tau)
$$

' $b$ ' has been evaluated using the solution of ' $x$ ' in (13) and equating the coefficient of $\sin (3 \tau)$ and $\cos (3 \tau), b=\alpha A^{3} / 4$. (22) Refer to (16) and (22) after using energy balance principle one gets,

$$
\alpha^{4} \frac{A^{10}}{64}+\alpha^{2} A^{6}-9 \alpha^{2} A^{4}+4 A^{2}-16=0 . \text { Hence } \mathrm{A}= \pm 2.395
$$

Now following the method of the previous section, it can be shown that,

$$
\alpha_{L}=\frac{1}{4 \pi} \frac{A^{2}}{4}\left(1+\frac{\left(3 \alpha A^{2}\right)^{2}}{4}+\frac{9\left(\alpha A^{2}\right)^{4}}{256}\right) \text { and } \alpha_{G}=\frac{1}{4 \pi}\left(1+\left(\frac{\alpha A^{2}}{4}\right)^{2}\right)
$$

Thus, it is seen that distortion increases the asymmetry in the shift of the poles around the imaginary axis of the complex frequency plane.

\section{Hard Self Excitation and Damping Factor}

The difference between the soft self excitation and hard self excitation $[5,6]$ arises because the choice of the operating point of an oscillator. For example, the expression of the equivalent damping can be written as:

$$
2 a_{e q}(A)=\left(G-a_{1}\right)-\frac{3}{4} a_{3} A^{2}-\frac{5}{8} a_{5} A^{4}
$$

It is to be noted that in a soft self excited oscillator, $a_{3}$ and $a_{5}$ are negative while $a_{1}$ is positive. But in a hard self excited oscillator it is found that $a_{1}$ and $a_{5}$ are positive and $a_{3}$ is negative. Unlike the soft-self excited oscillator in the hard-self excited oscillator, the oscillation can build up only from a finite initial excitation.

\section{Conclusion}

Pole-movement in oscillator, both soft-self and hard-self excited oscillators, has been looked into from new perspective and new results have been presented with experimental results in support. Modified Barkhausen Criterion has been proposed that takes into the non-steady and non-linear nature of the oscillator problems.

\section{Acknowledgments}

Authors are thankful to the management of Sir J.C. Bose School of Engineering for carrying out the work on Sir J.C. Bose Creativity Centre of SKF Group of Institutions, Mankundu, Hooghly-712 139, West Bengal, INDIA.

\section{References}

1. E. Vidal, A. Poreda and M. Ismail, "Describing functions and oscillators", IEEE Circuits and Devices, vol.17, No 6, pp 711, November 2009.

2. A. Gelb and W. Vader Velde, "Multiple input describing function and nonlinear system design", McGraw Hill 1968.

3. E. Lindberg, "Oscillators - An Approach for a Better Understanding", Proceedings of the 2003 European Conference on Circuit Theory and Design, Krakow, Poland, 2003.

4. B. N. Biswas, "Phase-lock theories and applications", Oxford and IBH, 1988.

5. Guillermo Gonzalez, "Foundations of Oscillator Circuit Design", ISBN 978-1-59693-163-3; Copyright 2006; Pages: 500.

6. Fan He, R Ribas Cyril Labuee and M Jezequel, "Discussion on the general oscillation start-up condition and Barkhausen Criterion", Analogue Integer Circ Sig Process, Springer Published online 13 December 2008.

7. Basab Chattterjee, B. N. Biswas "Performance Analysis of the 2nd Order Phase Lock Loop when Phase Modulation is Added to the Voltage Controlled Oscillator", IETE Journal of Research, vol. 56, Issue 6, pp 346, Nov-Dec, 2010.

8. B.N. Biswas, S. Pal, S. Chatterjee, "Movement of Poles in Oscillator", Proceeding of E2NC 2011, pp. 155- 161, Jan. 2011 